\title{
Medication Adherence and Patients Satisfaction among Psychiatric Outpatients in a Rural Nigerian Tertiary Healthcare Facility
}

Joseph O Fadare*, Musiliu A Lawal, Adetokunbo O Elegbede, David O Joseph, Bolawale A Ampitan

Majekodunmi L Ayodele

Department of Pharmacology, Ekiti State University, Ado-Ekiti, Nigeria

${ }^{*}$ Corresponding author: Joseph O Fadare, Department of Pharmacology, Ekiti State University, Ado-Ekiti, Nigeria, Tel: +234-8138048127; E-mail: mailto:jofadare@gmail.com

Received date: March 25, 2014, Accepted date: May19, 2014, published date: May 25, 2014

Copyright: (c) 2014 Joseph O Fadare, et al. This is an open-access article distributed under the terms of the Creative Commons Attribution License, which permits unrestricted use, distribution, and reproduction in any medium, provided the original author and source are credited.

\begin{abstract}
Introduction

Adherence in mental disorders is a bit different from that in other medical conditions because of some peculiarities associated with these disorders. A major factor that may contribute to non-adherence is the satisfaction or otherwise of the patients to the treatment being received from the health care provider. The main objective of this study was to determine the level of adherence and treatment satisfaction among psychiatric patients in a rural healthcare facility in South-West Nigeria.
\end{abstract}

\section{Methods}

This cross-sectional study used the 8-item Morisky Medication Adherence Scale (MMAS-8) and the Treatment Satisfaction Questionnaire for Medication (TSQM 1.4) to assess the adherence and patient satisfaction respectively.

\section{Results}

A total of 100 patients participated in the study out of which $57(57 \%)$ were males and $43(43 . \%)$ female with a mean age was $37 \pm 12$.8years. Schizophrenia was the most common diagnosis $(67 \%)$ found among the study participants. Forty-five $(45.9 \%)$ patients scored high for adherence, $34(34.7 \%)$ had medium score while $19(20.4 \%)$ scored low. Among patients with schizophrenia, $23(39 \%)$ had high adherence, $26(44.1 \%)$ medium and $10(16.9 \%)$ low adherence. The scoring of the Treatment Satisfaction Questionnaire administered to the patients was done according to the interpretation key. The mean Effectiveness Score was $74.3 \pm 15.6$, Side-Effects $(86.6 \pm 24.4)$, Convenience $(73.1 \pm 12.8)$ and Satisfaction $(73.5 \pm 15.7)$. The mean satisfaction score of patients with schizophrenia was $73.5 \pm 14.8$ while the score for all other diagnoses (grouped as one) was $74.4 \pm 16.5$.

\section{Conclusion}

Medication Adherence and Treatment Satisfaction among psychiatric outpatients in a rural Nigerian hospital is good. There is however a need to sustain and even improve on this outcome through better therapeutic alliance with patients and education on the nature of adverse drug reactions to prescribed drugs.

Keywords: Medication compliance; Mental disorders; Psychiatric patients

\section{Introduction}

Adherence to a medication can be defined as the extent to which a patient follows instructions given by a physician regarding use of the prescribed medication [1]. Adherence in mental disorders is a bit different from that in other medical conditions because of some peculiarities; most mental conditions are chronic with periods of exacerbation and relapse, poor insight in conditions like schizophrenia and associated cognitive problems[2,3]. Factors that have been identified to affect adherence among psychiatric patients include living alone, fear of adverse drug reactions, perceived inefficacy of the medication, using potentially inappropriate medications and the belief that the medication is no longer needed [4,5]. The employment status of patients, level of social functioning and substance abuse are other predictors of non-adherence [6]. The consequences of non-adherence among patients with mental disorders include higher healthcare costs, frequent hospital admissions and increased mortality [7]. Several studies have reported on adherence among various categories of psychiatric patients; in a study among Palestinians patients only about $22 \%$ had a high level of adherence while two separate Nigerian studies also correlated a similar level of poor adherence [8-10]. A major factor that may contribute to non-adherence is the satisfaction or otherwise of the patients to the treatment being received from the health care provider. This relationship between adherence and treatment satisfaction has not been well explored in previous Nigerian studies. Also, the above cited Nigerian studies were conducted in mental health facilities that are located in urban centers of the country and might not have reflected the true situation in a rural Nigerian setting. The main objective of this study was to determine the level of adherence to medications among psychiatric outpatients and factors that may affect it in a rural healthcare facility in South-West Nigeria. A secondary objective of the study was to determine the level of treatment satisfaction of the patients and to investigate the relationship between the two factors 
Page 2 of 5

\section{Methods}

\section{Setting}

The study was carried out at the outpatients' clinics of the Mental Health Department of Federal Medical Centre, Ido-Ekiti, South-West Nigeria from January 1 to June 30, 2013. It is a tertiary healthcare facility that caters for the secondary and tertiary care needs of the people of Ekiti and neighbouring states in the south-western part of Nigeria. The facility is adequately staffed with six consultants, 12 psychiatry residents, 32 psychiatry nurses and 6 psychologists. The department runs two outpatient clinics weekly with an estimated weekly patient load of 40-60. Patients are usually given clinic appointments bi-weekly or monthly depending on how stable their condition is.

\section{Study population}

Consecutive patients with psychiatric conditions attending the adult psychiatry outpatients' clinic of the hospital were invited to participate in the study after signing the informed consent document. The inclusion criteria for the study were: patients aged 14 years and above, those who had been on medications for at least three months and those that agreed to participate in the study. Patients who refused to participate in the study, those on medications for less than three months and those with severe acute symptomatology were excluded from the study. The investigators ensured that there was no discrimination against those that refused participation in the study by attending to them promptly and professionally. The decision to use three months of medication use as the cut-off for inclusion into the study was due to the fact that most patients had 2-4 weekly appointments and would have had 3 - 6 clinic appointments. Previous studies on adherence conducted in Nigeria and Ethiopia had used six and one clinic attendances as cut off point $[11,12]$.

\section{Sample size}

The sample size needed for this study was calculated using the formula for descriptive studies:

\author{
Sample size $(\mathrm{n})=\left[\operatorname{DEFF}^{*} \mathrm{~Np}(1-\mathrm{p})\right] /\left[\left(\mathrm{d} 2 / \mathrm{Z} 21-\alpha / 2^{*}(\mathrm{~N}-1)+\mathrm{p}^{*}(1-\mathrm{p})\right]\right.$ \\ Where, $\mathrm{N}=$ Population size (800) \\ $\mathrm{p}=$ prevalence of poor adherence $(0.4)$ \\ $\mathrm{d}=$ Precision $(0.1)$ \\ DEFF=Design effect (1.5) \\ $\mathrm{Z} 1-\alpha / 2=1.96$
}

The hypothesized prevalence of poor adherence was based on results from two similar studies in Nigeria [9,11]. An estimated minimum sample size of 83 was obtained using the above mentioned assumptions, however one hundred patients were recruited to give better power and accommodate for potential drop outs.

\section{Method}

This was a cross-sectional study that included a structured patient interview and administration of two validated instruments by two research assistants (psychiatry residents). The patients were intimated with the objectives of the study during their routine clinic visit and invited to participate in the study. The interviews were conducted in the consulting rooms of the department after the patient had been attended to in the clinic and this guaranteed privacy for the respondents.

\section{Study instruments}

The validated self-assessing adherence tool (The 8-item Morisky Medication Adherence Scale (MMAS-8) which has been previously used in Nigeria studies was administered $[9,13]$. This instrument is a form of self-report of medication adherence by patients and it is made up of 8 questions. The MMAS- 8 collects information on how frequent patients may forget to take their medications and the reasons for this using a binary (Yes/No) and a five option response version (never/ rarely/sometimes/often/always). The summary of the scoring interpretation is as follows: 0 (for high adherence), 1-2 (medium adherence) and $>2$ (low adherence). An additional questionnaire for collection of socio-demographic details, diagnoses list of prescribed medications and factors that may affect adherence was also administered. .Diagnosis was made clinically and using appropriate diagnostic instruments and classified according to the International Classification of Disease (ICD 10). Treatment satisfaction was assessed using the Treatment Satisfaction Questionnaire for Medication (TSQM 1.4) [14,15].The TSQM Version 1.4 is a 14 -item validated instrument made up of four domains. The four domains of the TSQM are the effectiveness domain (questions 1-3), side effects domain (questions 4-8), convenience domain (questions 9-11) and the global satisfaction domain (questions 12-14). The TSQM 1.4 domains were scored according to the recommendation of the authors and it ranges from 0 to 100 with the higher scores reflecting higher satisfaction in that particular domain. The questionnaires were administered by medical doctors during the clinic visit once informed consent is obtained.

\section{Statistical analysis}

The information obtained from the general questionnaire was coded and entered using IBM SPSS version 19 (IBM Corporation, Armonk, NY, USA). The information from the remaining two instruments (MMAS-8 and TSQM 1.4) was summarized using their interpretation keys and entered using the same version of IBM SPSS software. The general characteristics of the patients were analyzed using descriptive statistics. Demographic variables, which were normally distributed, were described as mean and standard deviation. The median and range were calculated for other demographic variables that were not normally distributed. Categorical variables are reported as frequency distribution and proportions with 95\% confidence intervals and were compared using the chi-square test or Fisher's exact test. One way ANOVA test was used to determine association between medication adherence level and the mean of all spheres of treatment satisfaction. Association between adherence level, employment status (unemployed/employed), level of education (below secondary education/ at least secondary education), monthly income (less than/above 20,000 Nigerian Naira), age and sex was explored using binary logistic regression (Odds Ratios).A P value of $<0.05$ was considered statistically significant.

\section{Ethical consideration}

Informed consent (verbal/written) was obtained from individual patients before being recruited into the study. The reason for including oral form of consent in this study is because of cultural issues raised by signing documents in developing nations of Africa and Asia. Ethical 
Citation: Fadare JO, Lawal MA, Elegbede AO, Joseph DO, Ampitan BA, Ayodele ML (2014) Medication Adherence and Patients Satisfaction among Psychiatric Outpatients in a Rural Nigerian Tertiary Healthcare Facility. J Psychiatry 17: 125. doi:10.4172/2378-5756.1000125

Page 3 of 5

approval was obtained from the Research Ethics Committee of the hospital before commencement of the study

Results

A total of 100 patients participated in the study out of which 57 (57\%) were males and 43 (43\%) female. The mean age was $37 \pm 12.8$ with the youngest patient being 14 years old while the oldest was 78 years.

\begin{tabular}{|c|c|}
\hline Variable & Frequency $(\%)$ \\
\hline \multicolumn{2}{|l|}{ Level of education } \\
\hline No education & $5(5.3)$ \\
\hline Primary education & $11(11.6)$ \\
\hline Secondary education & $32(33.7)$ \\
\hline Tertiary education & $47(49.5)$ \\
\hline \multicolumn{2}{|l|}{ Occupational status } \\
\hline Unemployed & $36(45)$ \\
\hline Trading & $16(20)$ \\
\hline Artisan & $7(8.8)$ \\
\hline Civil servants & $18(22.5)$ \\
\hline Professional & $3(3.8)$ \\
\hline \multicolumn{2}{|l|}{ Monthly income } \\
\hline Less than 20,000 Naira (125 USD) & $37(60.7)$ \\
\hline $20,000-50,000$ Naira (125-303 USD & $17(27.9)$ \\
\hline 50,000 - 100,000 Naira (303-606 USD) & $7(11.5)$ \\
\hline \multicolumn{2}{|l|}{ Age groups } \\
\hline $14-39$ years & $62(66.7)$ \\
\hline $40-59$ years & $24(25.8)$ \\
\hline$>60$ years & $7(7.5)$ \\
\hline
\end{tabular}

Table 1: Socio-demographic details of patients

Table 1 shows the distribution of patients according to sex, duration of illness, educational, employment and income earning status. Schizophrenia was the most common diagnosis (67\%) found among the study participants. Figure 1 shows the detailed analysis of the diagnoses.

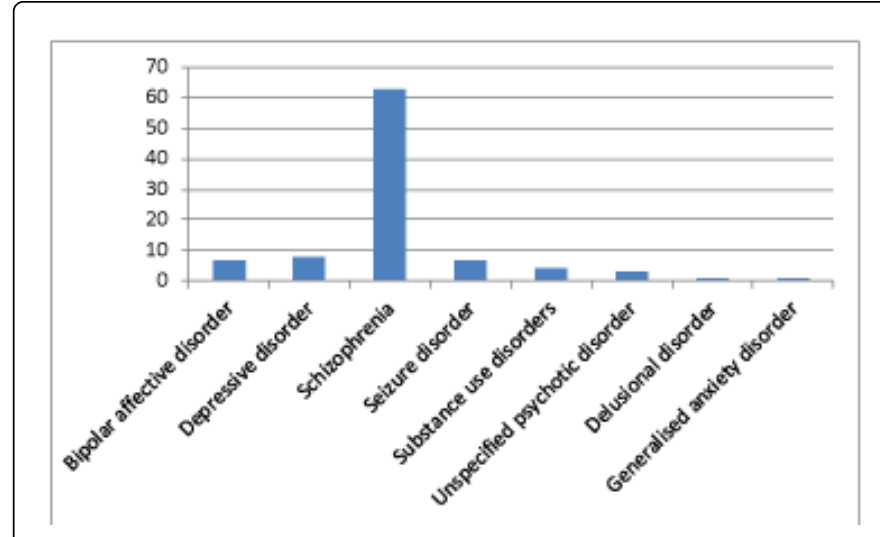

Figure 1: Diagnoses of the patients

The anti-muscarinic drug, tri-hexyphenidyl was the most commonly prescribed drug (26.3\%), followed by the anti-psychotic, Haloperidol (17.6\%). The details of prescribed drugs are as listed on Table 2. The total number of drugs prescribed for the participants during the study period was 232 with a mean of $2.3 \pm 1$. Twenty five patients had only one drug prescribed while only one patient was placed on six medications. The detailed information about the number of drugs prescribed per patient is shown on Figure 2.

\begin{tabular}{|l|l|}
\hline Drug Prescribed & Frequency (\%) \\
\hline Trihexyphenidyl (Benzhexol) & $54(26.3)$ \\
\hline Haloperidol & $36(17.6)$ \\
\hline Chlorpromazine & $29(14.1)$ \\
\hline Carbamazepine & $17(8.3)$ \\
\hline Fluphenazine & $16(7.8)$ \\
\hline Trifluoperazine & $18(8.8)$ \\
\hline Olanzapine & $12(5.9)$ \\
\hline Amitryptiline & $15(7.5)$ \\
\hline Risperidone & $5(2.4)$ \\
\hline Fluoxetine & $1(0.5)$ \\
\hline Clozapine & $1(0.5)$ \\
\hline Nitrazepam & $1(0.5)$ \\
\hline
\end{tabular}

Table 2:Prescribed drugs for psychiatric disorders 
Page 4 of 5

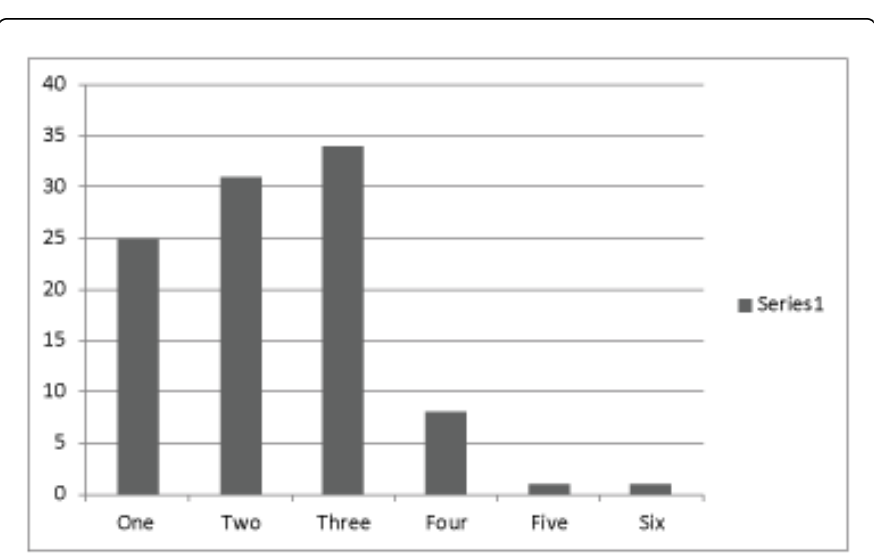

Figure 2: Number of drugs prescribed per patient

Forty-five $(45.9 \%)$ patients scored high for adherence, $34(34.7 \%)$ had medium score while 19 (20.4\%) scored low. Table 2 shows the relationship between adherence and some socio-demographic characteristics of the participants. The association between adherence,, monthly income, employment status, sex and educational level is as shown in table.The scoring of the Treatment Satisfaction Questionnaire administered to the patients was done according to the interpretation key. The mean Effectiveness Score was $74.3 \pm 15.6$, SideEffects $(86.6 \pm 24.4)$, Convenience $(73.1 \pm 12.8)$ and Satisfaction (73.5 $\pm 15.7)$. Table 2 shows the comparison of the means of the different spheres of the Treatment Satisfaction Questionnaire with the 3 categories of medication adherence. The mean satisfaction score of patients with schizophrenia was $73.5 \pm 14.8$ while the score for all other diagnoses (grouped as one) was $74.4 \pm 16.5$. The difference between these two groups was not statistically significant $(P>0.05)$. The patients also identified some factors that may affect adherence to their medications and they include inadequate information (54.8\%), prolonged duration of drug use (14.9\%), lack of family support (14.9\%), occurrence of adverse effects (13.5\%) and cost (13.5\%). The median total monthly cost of accessing treatment (medications, transportation and consultation fees) of patients in this study was 2250 Naira (13.6 USD) with drugs and transportation being responsible for about $57.3 \%$ and $32.3 \%$ of the total cost respectively. The relationship between medication adherence, number of prescribed drugs and monthly cost of medications is shown in figures.

Discussion

The main objective of this study was to assess the level of medication adherence among psychiatric patients and its relationship with treatment satisfaction. There was a male preponderance (57\%) in this study, in keeping with findings from similar studies in developing countries $[16,17]$. The median duration for seeking care among this cohort of patients was less than that found in another Nigerian study [18], a difference that could be explained by the larger sample size and community -based nature of that study. A significant number among this cohort were unemployed as at the time the study was conducted. Unemployment has been identified as a common denominator among mentally ill patients in Nigeria $[19,20]$. This association could be bidirectional; mental illness and its associated stigma could lead to unemployment or the patient's condition could lead to job loss. The fact that over $60 \%$ of the patients earned less than $20,000 \mathrm{Naira}(=125$ USD) monthly reflects the link between poverty and mental disorders [21].
Only $20.4 \%$ of patients in this study were found to have low medication adherence in contrast to $48 \%$ found in a previous Nigerian study looking at general psychiatric outpatients in Nigeria using the same instrument [9]. The difference between this study and ours may be due to the larger sample size and the urban setting where it was carried out. In a similar study on anti-psychotic drug adherence in Palestine, $33.6 \%, 44.3 \%$ and $22.1 \%$ were with low, medium and high levels of adherence respectively [8]. The formulation of the drugs or route of administration has also been implicated in non-adherence [22], however there was no significant difference in the adherence scores between patients who had long-acting injectables and the general patient population. Also, adherence can be influenced by the generation of drugs being used (first versus second generation antipsychotics) [23]; this relationship could not be fully explored in our study because of the relatively small number of patients who had newer anti-psychotics prescribed. Among other factors that could affect adherence, inadequate information about disease condition and its treatment was common among our study participants. Good social support, fewer number of medication, cost of treatment and fear of adverse drug reactions are factors that have been identified in other studies $[5,11,24]$.

The means of the different domains of patient satisfaction found in our study were higher than those found by Sweileh and co-workers [8]. In that study, the mean scores for the different domains were Effectiveness (72.6 \pm 20.5$)$, Side-Effects (67.9 \pm 31.5$)$, Convenience $(63.2 \pm 14.3)$ and Global Satisfaction $(63.1 \pm 15.8)$. Some workers have suggested that the development of a therapeutic alliance between the patients and the managing team could lead to better medication adherence [25]. There is also another school of thought that believes that focus on treatment related attitudes will help to promote adherence more than the earlier concept of global insight into the illness [2].

\section{Study Limitations}

The sample size and the fact that the study was conducted in one centre were major limitations of the study. Cases of missing values also could have some impact on the interpretation of the results of this study. Though the study used an interviewer administered questionnaire, respondents sometimes refused to answer some sensitive information like income level or even age because of fear about possible use of their information for tax related or other official purposes. Though the instruments used for the study relied on selfreported information that could have some kind of bias or inaccurate report, the standardized and validated nature of the data collection instruments used in the study is the major strength of the study.

\section{Conclusion}

Medication adherence and treatment satisfaction among psychiatric outpatients in a rural Nigerian hospital is satisfactory. There is however a need to sustain and even improves on this outcome through better therapeutic alliance with patients and education on the nature of adverse drug reactions to prescribed drugs.

\section{Acknowledgements}

The authors are grateful to the Mental Health Department of the Federal Medical Centre, Ido-Ekiti, South-West Nigeria for allowing the study to take place in their centre. We are also very grateful to the 
Citation: Fadare JO, Lawal MA, Elegbede AO, Joseph DO, Ampitan BA, Ayodele ML (2014) Medication Adherence and Patients Satisfaction among Psychiatric Outpatients in a Rural Nigerian Tertiary Healthcare Facility. J Psychiatry 17: 125. doi:10.4172/2378-5756.1000125

Page 5 of 5

resident doctors, nurses and medical records staff who contributed to the successful completion of the study.

Conflict of Interest: None disclosed

\section{References}

1. $\quad$ Osterberg L, Blaschke T (2005) Adherence to medication. N Engl J Med 353: 487-97.

2. Beck EM, Cavelti M, Kvrgic S, Kleim B, Vauth R (2011) Are we addressing the 'right stuff' to enhance adherence in schizophrenia? Understanding the role of insight and attitudes towards medication. Schizophr Res 132: 42-9.

3. Campbell NL, Boustani MA, Skopelja EN, Gao S, Unverzagt FW,et al. (2012) Medication adherence in older adults with cognitive impairment: a systematic evidence-based review. Am J Geriatr Pharmacother 10: 165-77.

4. Campbell NL, Dexter P, Perkins AJ, Gao S, Li L, Skaar TC, et al. (2013) Medication adherence and tolerability of Alzheimer's disease medications: study protocol for a randomized controlled trial. Trials 14: 125.

5. Tamburrino MB, Nagel RW, Chahal MK, Lynch DJ (2009) Antidepressant medication adherence: a study of primary care patients. Prim Care Companion J Clin Psychiatry 11: 205-11.

6. Breen R, Thornhill JT (1998) Noncompliance with medication for psychiatric disorders. CNS Drugs 9:457-71.

7. Boden R, Brandt L, Kieler H, Andersen M, Reutfors J (2011) Early nonadherence to medication and other risk factors for rehospitalization in schizophrenia and schizoaffective disorder. Schizophr Res133: 36-41.

8. Sweileh WM, Ihbesheh MS, Jarar IS, Sawalha AF, Abu Taha AS, et al. (2012) Antipsychotic medication adherence and satisfaction among Palestinian people with schizophrenia. Curr Clin Pharmacolb 7: 49-55.

9. Adewuya AO, Owoeye OA, Erinfolami AR, Coker AO, Ogun OC, et al. (2009) Prevalence and correlates of poor medication adherence amongst psychiatric outpatients in southwestern Nigeria. Gen Hosp Psychiatry 31: 167-74.

10. James BO, Omoaregba JO (2011) Prevalence and predictors of poor medication adherence among out-patients at a psychiatric hospital in Benin City, Nigeria. Int J Psychiatry Clin Pract 15: 27-34.

11. Adelufosi AO, Adebowale TO, Abayomi O, Mosanya JT (2012) Medication adherence and quality of life among Nigerian outpatients with schizophrenia. Gen Hosp Psychiatry34: 72-9.

12. Tesfay K, Girma E, Negash A, Tesfaye M, Dehning S (2013) Medication non-adherence among adult psychiatric out patients in Jimma University Specialized Hospital, Southwest Ethiopia. Ethiop J Health Sci 23: 227-36.
13. Morisky DE, Ang A, Krousel-Wood M, Ward HJ (2208) Predictive validity of a medication adherence measure in an outpatient setting. J Clin Hypertens (Greenwich) 10: 348-54.

14. Atkinson MJ, Sinha A, Hass SL, Colman SS, Kumar RN, Brod M, et al. (2004) Validation of a general measure of treatment satisfaction, the Treatment Satisfaction Questionnaire for Medication (TSQM), using a national panel study of chronic disease. Health Qual Life Outcomes 2:12.

15. Atkinson MJ, Kumar R, Cappelleri JC, Hass SL (2005) Hierarchical construct validity of the treatment satisfaction questionnaire for medication (TSQM version II) among outpatient pharmacy consumers. Value Health. 8: S9-S24.

16. Banerjee I, Roy B, Sathian B, Chakraborty PK, Saha A (2013) Socio demographic profile and utilization pattern of antipsychotic drugs among schizophrenic inpatients: a cross sectional study from western region of Nepal. BMC Psychiatry 13: 96.

17. Ponto T, Ismail NI, Abdul Majeed AB, Marmaya NH, Zakaria ZA (2010) A prospective study on the pattern of medication use for schizophrenia in the outpatient pharmacy department, Hospital Tengku Ampuan Rahimah, Selangor, Malaysia. Methods Find Exp Clin Pharmacol 32: 427-32.

18. Gureje O, Uwakwe R, Udofia O, Wakil A, Adeyemi O, Enyidah N (2008) Common psychiatric disorders over a lifetime: age of onset, risk and treatment contact in the Nigerian survey of mental health and wellbeing. Afr J Med Med Sci 37: 207-17.

19. Amoran OE, Lawoyin TO, Oni OO (2005) Risk factors associated with mental illness in Oyo State, Nigeria: a community based study. Ann Gen Psychiatry 4: 19.

20. Gbiri CA, Badru FA, Ladapo HT, Gbiri AA (2011) Socio-economic correlates of relapsed patients admitted in a Nigerian mental health institution. Int J Psychiatry Clin Pract 15: 19-26.

21. Patel V, Kleinman A (2003) Poverty and common mental disorders in developing countries. Bull World Health Organ 81: 609-15.

22. Cecchi C, Canonico PL (2012) Pharmaceutical formulations and adherence to pharmacological treatment in psychiatry: the example of oral disintegrating tablet of olanzapine. Riv Psichiatr 47: 30-9.

23. Chang CM, Wu KY, Liang HY, Wu EC, Chen CY, et al. (2012) Adherence patterns with first- versus second-generation antipsychotics for newly diagnosed schizophrenia in Taiwan. Psychiatr Serv 63: 504-7.

24. Sansone RA, Sansone LA (2012) Antidepressant adherence: are patients taking their medications? Innov Clin Neurosci 9: 41-6.

25. Charpentier A, Goudemand M, Thomas P (2009) Therapeutic alliance, a stake in schizophrenia. Encephale 35: 80-9. 